PREPARED FOR THE U.S. DEPARTMENT OF ENERGY, UNDER CONTRACT DE-AC02-76CH03073

PPPL-3955

PPPL-3955

UC-70

Phenomenology of Compressional Alfvén Eigenmodes

by

E.D. Fredrickson, N.N. Gorelenkov, and J. Menard

May 2004

$\left.\stackrel{M}{M}\right|_{\substack{\text { PRInCETON PLASIMA } \\ \text { PHYSICS LABORATORY }}} ^{D}$

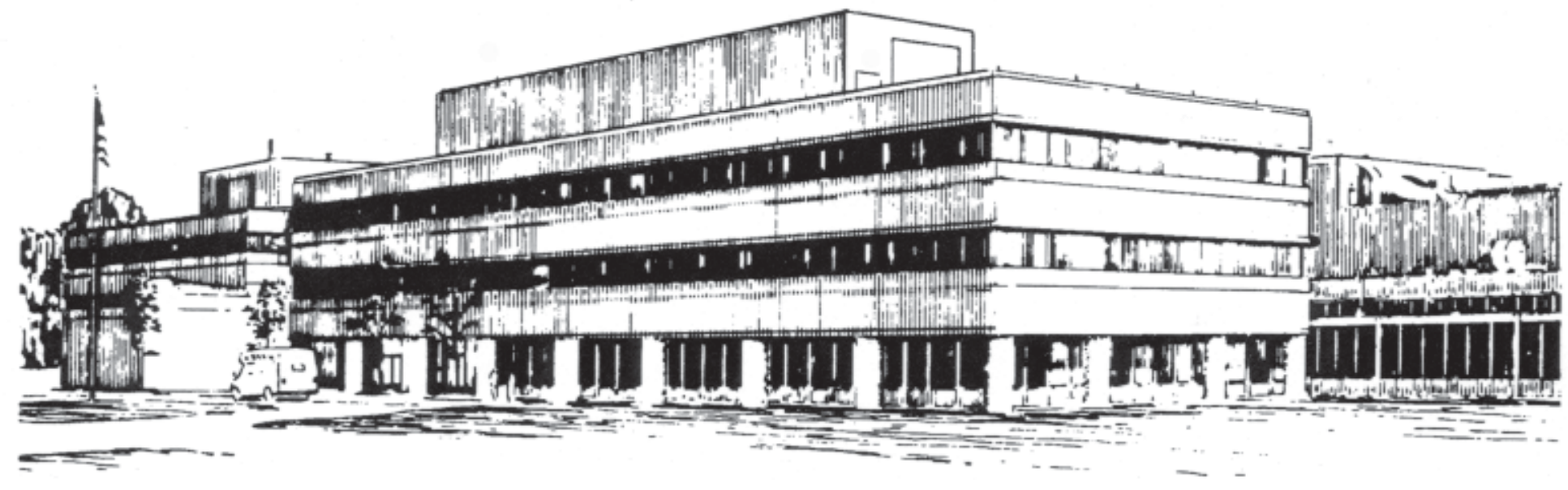

PRINCETON PLASMA PHYSICS LABORATORY PRINCETON UNIVERSITY, PRINCETON, NEW JERSEY 


\section{PPPL Reports Disclaimer}

This report was prepared as an account of work sponsored by an agency of the United States Government. Neither the United States Government nor any agency thereof, nor any of their employees, makes any warranty, express or implied, or assumes any legal liability or responsibility for the accuracy, completeness, or usefulness of any information, apparatus, product, or process disclosed, or represents that its use would not infringe privately owned rights. Reference herein to any specific commercial product, process, or service by trade name, trademark, manufacturer, or otherwise, does not necessarily constitute or imply its endorsement, recommendation, or favoring by the United States Government or any agency thereof. The views and opinions of authors expressed herein do not necessarily state or reflect those of the United States Government or any agency thereof.

\section{Availability}

This report is posted on the U.S. Department of Energy's Princeton Plasma Physics Laboratory Publications and Reports web site in Fiscal Year 2004. The home page for PPPL Reports and Publications is: http://www.pppl.gov/pub_report/

DOE and DOE Contractors can obtain copies of this report from:

U.S. Department of Energy

Office of Scientific and Technical Information

DOE Technical Information Services (DTIS)

P.O. Box 62

Oak Ridge, TN 37831

Telephone: (865) 576-8401

Fax: (865) 576-5728

Email: reports@adonis.osti.gov

This report is available to the general public from:

National Technical Information Service

U.S. Department of Commerce

5285 Port Royal Road

Springfield, VA 22161

Telephone: $1-800-553-6847$ or

(703) $605-6000$

Fax: (703) 321-8547

Internet: http://www.ntis.gov/ordering.htm 


\title{
Phenomenology of compressional Alfvén eigenmodes
}

\author{
E D Fredrickson, N N Gorelenkov, J Menard \\ Princeton Plasma Physics Laboratory, Princeton, New Jersey 0854
}

\begin{abstract}
Coherent oscillations with frequency $0.3 \leq \omega / \omega_{\mathrm{ci}} \leq 1$, are seen in the National Spherical Torus Experiment [M. Ono, S M Kaye, Y-K M Peng, et al., Nucl. Fusion 40, 557 (2000)]. This paper presents new data and analysis comparing characteristics of the observed modes to the model of compressional Alfvén eigenmodes (CAE). The toroidal mode number has been measured and is typically between $7<\mathrm{n}<9$. The polarization of the modes, measured using an array of four Mirnov coils, is found to be compressional. The frequency scaling of the modes agrees with the predictions of a numerical 2-D code, but the detailed structure of the spectrum is not captured with the simple model. The fast ion distribution function, as calculated with the beam deposition code in TRANSP [R. V. Budny, Nucl. Fusion 34, 1247 (1994)], is shown to be qualitatively consistent with the constraints of the Doppler-shifted cyclotron resonance drive model. This model also predicts the observed scaling of the low frequency limit for CAE.
\end{abstract}




\section{INTRODUCTION}

The study of fast ion instabilities in conventional aspect ratio tokamaks is motivated in large part by their potential to negatively impact the ignition threshold in fusion reactors by causing fast ion losses. Spherical tokamak (ST) reactors, with intrinsically low magnetic fields, are particularly susceptible to fast ion driven instabilities. The 3.5 MeV alpha particles from the D-T fusion reaction in proposed ST reactors will have velocities much higher than the Alfvén speed. The Larmor radius of the fusion alphas, normalized to the plasma size, will also be larger than for conventional aspect ratio tokamak reactors. The resulting longer wavelengths of the Alfvénic instabilities will be more effective in driving fast ion loss [1]. It is important to extend the predictive capabilities for kinetic instabilities, as developed on conventional aspect ratio tokamaks, to low aspect ratio and to benchmark the results on present ST devices.

The National Spherical Torus Experiment (NSTX) [2] is a low aspect ratio $(\mathrm{R} / \mathrm{a} \approx 1.3)$ toroidal device well suited to study fast ion instabilities in a parameter regime relevant to an $\mathrm{ST}$ reactor. The plasma major and minor radii are $\approx 0.8 \mathrm{~m}$ and $\approx 0.65 \mathrm{~m}$, respectively. Some representative operational parameters from the experiment described here, are: $\approx 0.9$ MA of plasma current, 3 to $6 \mathrm{kG}$ toroidal field, central electron density is $2.4-3.9 \times 10^{19} / \mathrm{m}^{3}$, and central electron temperature of $0.5-0.9 \mathrm{keV}$. The extremes of these parameters are not, of course, reached simultaneously. The plasmas are heated with 0.52 to $2.1 \mathrm{MW}$ of deuterium neutral beam injection (NBI) power with an acceleration voltage of between 49 and $65 \mathrm{kV}$. The full-energy neutral beam ions in these experiments have velocities ranging from 1.3 - 3.2 times the central Alfvén velocity. The dimensionless parameters of beam ions in NSTX are similar to those of fusion $\alpha$ 's in 
proposed ST reactors, with the caveat that the fusion $\alpha$ distribution will be more isotropic in velocity space and will not have the half and third energy components of beam ion distributions.

Neutral beam heated plasmas in NSTX typically have a wide range of fast ion driven instabilities extending from fishbone-like modes at frequencies of 10's of $\mathrm{kHz}$ [35] through the toroidal Alfvén eigenmodes (TAE) at frequencies of order $100 \mathrm{kHz}$ [6-9] and up to Compressional or Global Alfvén eigenmode (CAE or GAE) frequencies of over $1 \mathrm{MHz}[10-16]$. The TAE activity appears to be similar to TAE observed in conventional aspect ratio tokamaks [9]. The fishbone modes have significant differences, and, although the Ion Cyclotron Emission (ICE) observed in conventional aspect ratio tokamaks [17-21] may be related to compressional Alfvén waves, the strong, ubiquitous CAE activity seen in NBI heated NSTX plasmas is, thus far, unique to low aspect ratio.

Enhanced fast ion losses have been correlated with both the TAE-like and fishbonelike modes [22], but there is no observed degradation in performance correlated with the appearance of CAE activity. There are some indications that the CAE do affect the fast ion distribution. Bursts of CAE activity in some cases appear to trigger the growth of the lower frequency fast ion driven instabilities, explainable by CAE-induced transport of fast ions in either real or velocity space.

This paper presents new data and analysis on the nature of the compressional Alfvén eigenmodes. In Sect. II a simple model for the CAE dispersion relation is reviewed and a numerical code, which calculates the eigenfunctions and finds the eigenfrequencies for $\mathrm{CAE}$, is described. The qualitative predictions of this simple model are compared to 
experimental data in Sects. III and IV. In Sect. III an experiment exploring the onset threshold scaling of CAE is described and in Sect. IV the data is analyzed and compared to predictions of CAE theory.

\section{Numerical modeling of the Compressional Alfvén mode eigenfunction}

Theory predicts that compressional Alfvén modes in low aspect ratio tokamaks are localized towards the outboard midplane in a two-dimensional (radial and poloidal) well [13]. The basic physics of these trapped CAE can be captured with a relatively simple set of equations in quasi-cylindrical geometry. The degree of localization and the radius of the peak mode amplitude can be estimated by solving the set of second order partial differential equations describing the compressional Alfvén wave dispersion relation. Approximate analytic solutions to this equation for model profiles were described previous work [13]; in this work approximate numerical solutions are found for the measured density profiles. The measured electron density and the inferred magnetic geometry are used to construct the 2-D dependence of $V_{\text {Alfvén }}$, an approximate, but separable, form of the "potential well" is found and the equations are then numerically integrated to find the eigenvalues (frequency and poloidal mode number).

The derivation for the 2-D well equations begins with the low beta dispersion relation for Alfvén waves.

$$
\frac{\partial^{2}}{\partial t^{2}} \vec{E}=-\omega^{2} \vec{E}=V_{A l f v e ́ n}^{2} \nabla^{2} \vec{E}
$$

The local electron $\beta_{\text {tor }}$ at the half radius (near the predicted peak in the wave amplitude) ranges from $0.3 \%$ to $3.3 \%$ for the data against which the code was benchmarked. The range of ion thermal $\beta$ 's for this experiment are comparable. This $\beta$ is low enough so that 
finite $\beta$ corrections to the $\mathrm{CAE}$ dispersion relation (coupling to the sound wave branch) may be neglected.

We will neglect the toroidal coupling terms and simplify the geometry to a quasicylindrical coordinate system. To retain the low aspect ratio physics of compressional Alfvén modes, i.e., the radial and poloidal localization, the Alfvén velocity dependence on both the minor radius and poloidal angle will be kept.

$$
\left\{r \frac{\partial}{\partial r} r \frac{\partial}{\partial r}+\frac{\partial^{2}}{\partial \theta^{2}}+\frac{r^{2}}{R^{2}} \frac{\partial^{2}}{\partial \vartheta^{2}}+\frac{\omega^{2}}{V_{\text {Alfvén }}^{2}} r^{2}\right\} f=0, \quad v(r, \theta)=\frac{\omega^{2}}{V_{\text {Alfvén }}^{2}} r^{2}
$$

While the effective potential in this equation depends on both minor radius and poloidal angle in a complicated way, a similarly shaped well can be formed by approximating it as the sum of a term dependent only on radius and a second term which depends on poloidal angle, $v(r, \theta) \approx v_{r}(r)-v_{\theta}(\theta)$. The equation may then be solved by introducing constants of separation for the poloidal and toroidal dimensions, corresponding to the poloidal and toroidal mode numbers. The toroidal contribution is simple, the poloidal and radial eigenfunctions are found by solving the coupled equations:

$$
\frac{1}{f_{r}}\left\{r \frac{\partial}{\partial r} r \frac{\partial}{\partial r}+v_{r}(r)\right\} f_{r}=-\frac{1}{g_{\theta}}\left\{\frac{\partial^{2}}{\partial \theta^{2}}-v_{\theta}(\theta)\right\} g_{\theta}=m^{2}
$$

With this definition, in the cylindrical limit $v(\theta)=0$ and the solutions are $g_{\theta}=e^{ \pm i m \theta}$.

The radial eigenfunction is found by solving the following equation where the "radial potential well" is the original "radial well" evaluated on the outboard midplane.

$$
\left\{\frac{1}{r} \frac{\partial}{\partial r} r \frac{\partial}{\partial r}+\left[\frac{\omega^{2}}{V_{\text {Alfvén }}^{2}}-\left.\frac{n^{2}}{R^{2}}\right|_{\theta=0}-\frac{m^{2}}{r^{2}}\right\} f_{r}=0\right.
$$

A conducting wall boundary condition is used at $r=1.2 \mathrm{a}$.

The poloidal eigenfunction is found by solving the second part of Eq. 3 . 


$$
\frac{\partial^{2}}{\partial \theta^{2}} g_{\theta}-\mathrm{V}(\theta) g_{\theta}=0, \quad \mathrm{~V}(\theta) \equiv v_{\theta}(\theta)-m^{2}
$$

The poloidal well, $v(\theta)$ is taken from the 2-D well at the radius of the well minimum. The eigenvalues, $\mathrm{m}$, are found to depend only weakly on the detailed shape of this "potential well". A representative comparison of the 2-D well (calculated with the eigenvalues of frequency and poloidal mode number from the model well) and the model well used in Eq. 5 is shown in Fig. 1.

Equations 4 and 5 make a set of two coupled ordinary differential equations, which qualitatively describe the compressional Alfvén eigenmodes. The mapping to a circular cross-section is roughly compensated for by scaling the minor radius by $\kappa^{1 / 2}$ and the poloidal dimension (poloidal wavelength) by $\kappa$ (where $\kappa$ is the elongation). The equations are solved iteratively with a simple shooting code to find the eigenfunctions and frequencies. In Fig. 2 are shown a representative radial eigenfunction and the shape of the well on the outboard midplane. The radial eigenfunction peaks around a normalized minor radius of about 0.5 , although it is not particularly well localized. The poloidal eigenfunction shape is shown in Fig. 3, together with the poloidal well, $v(\theta)$ as in Eq. 3 .

\section{EXPERIMENTAL METHOD AND RESULTS}

Previous experiments have provided evidence that Compressional Alfvén Eigenmodes are beam driven and are natural plasma resonances, rather than being of the class of energetic particle modes [14,23]. It is then not surprising that previous experiments found a sharp threshold in beam energy (fast ion velocity) for excitation of the modes [24] and that the level of CAE activity becomes stronger with higher beam 
voltage. However, the threshold does not correspond to a simple $\mathrm{V}_{\text {beam }} / \mathrm{V}_{\text {Alfvén }}$ resonance as was the case for TAE modes, but is more complex.

The experiment described here elucidates this aspect of the CAE instability by empirically examining the threshold for excitation of CAE as a function of the ratio of the beam ion velocity to the Alfvén speed. The Alfvén speed was varied in a sequence of discharges by systematically stepping the toroidal field from $3 \mathrm{kG}$ to $6 \mathrm{kG}$ in $0.5 \mathrm{kG}$ steps. At each toroidal field, the beam voltage was scanned to determine the voltage threshold for exciting CAE activity. The beam power (fast ion $\beta$ ) resulting from a single source is strongly dependent on beam voltage, but experiments with multiple sources have found that the threshold voltage is only weakly dependent on power. The change in the beam voltage threshold over a factor of roughly two in toroidal field strength was less than $15 \%$.

A typical plasma shot from this experiment can be seen in Fig. 4. An $80 \mathrm{kV}$ beam source is injected from about $0.1 \mathrm{~s}$ to $0.2 \mathrm{~s}$ to aid in the plasma formation during the current ramp and early current flattop. A $20 \mathrm{~ms}$ ohmic period follows $(0.2-0.22 \mathrm{~s})$ to provide a background frame for the CHERS ion temperature diagnostic [25] (and to allow time for the $80 \mathrm{kV}$ beam ions to, at least partially, thermalize). One or two beam sources are then injected from 0.22 to $0.26 \mathrm{~s}$ with the voltage varied from shot to shot between $49 \mathrm{kV}$ and $65 \mathrm{kV}$. In some discharges a second $40 \mathrm{~ms}$ beam blip is injected (0.28 to $0.32 \mathrm{~s}$ ), again following a $20 \mathrm{~ms}$ ohmic period. As the density generally increases throughout the discharge, this provides some data on density scaling.

The CAE activity is detected with an array of high bandwidth (0 to $>3.5 \mathrm{MHz})$ Mirnov coils. Of particular interest is the array of three coils for measuring the toroidal 
mode number and a fourth coil, oriented to measure the toroidal magnetic fluctuation amplitude and phase, for determining the mode polarization (shear or compressional). The detected coherent magnetic fluctuation amplitudes vary from as low as a few $\mu \mathrm{G}$ up to a few $\mathrm{mG}$. The equilibrium diagnostics include electron density and temperature measurements with a Thomson scattering diagnostic taking profile data every $\approx 17 \mathrm{~ms}$ [26]. The ion temperature and impurity velocity are measured over $20 \mathrm{~ms}$ intervals with the CHERS diagnostic. The equilibrium magnetic configuration is inferred with the EFIT code [27,28].

In Fig. 5 are shown equilibrium data for a representative shot from this experiment. In Fig. 5a is shown the lower single null, diverted equilibrium shape with an effective elongation of $\approx 1.8$. The location of the plasma-limiting surface is indicated schematically by the solid black curve. The small black squares to the right of the plasma indicate the poloidal positions of the outer Mirnov coils. Figure 5b shows the Thomson scattering electron density profile from the magnetic axis to the plasma edge. In Figure $5 \mathrm{c}$ are shown the poloidal field and $\bmod [B]$ profiles as calculated with the EFIT code, which together with the density provides the experimental input needed to calculate the local Alfvén velocity (the dominant ionic plasma components are assumed to be deuterium and carbon, both with 2 A.M.U. per electron, fully stripped).

The range of $V_{\text {Alfvén }}$ and $V_{\text {beam }}$ for the data set of stable and unstable discharges is shown in Fig. 6. In this figure the full energy beam ion velocity is graphed vs. the central Alfvén velocity ( $V_{\text {Alfvén }}$ is generally higher towards the plasma edge). The dependence of the threshold on the Alfvén velocity is found to be weak, although the beam voltage threshold does appear to increase slightly with toroidal field. The dependence of the 
level of CAE activity on beam voltage, however, does indeed represent a threshold. The change in beam ion velocity from below the threshold where there is no detectable CAE activity, to a condition with strong CAE activity can be as small as 5\%, i.e., from $\approx 2.3 \times 10^{6}$ to $\approx 2.4 \times 10^{6} \mathrm{~m} / \mathrm{s}$ or $55-60 \mathrm{kV}$.

\section{ANALYSIS}

A spectrogram of representative Mirnov coil data is shown in Fig. 7. The CAE activity is seen between approximately $1.4 \mathrm{MHz}$ and $2 \mathrm{MHz}$. The modes are indicated by the bands, sweeping down by $\approx 250 \mathrm{kHz}$ in frequency during this period. The lower frequency bands tend to be somewhat broader in frequency, and more detailed analysis suggest that these bands consist of multiple modes of much the same amplitude and nearly degenerate in frequency. The higher frequency bands are narrower and appear to consist of a single, dominant eigenmode. The nearly vertical black bars at $\approx 0.222 \mathrm{~s}$ and $0.26 \mathrm{~s}$ are at present unidentified. The modes end very shortly after the termination of neutral beam injection at $0.26 \mathrm{~s}$.

The observed mode activity often, but not always, consists of multiple modes with a hierarchy of frequency separation. In Fig. 7 the spacing of the dominant peaks is about $75 \mathrm{kHz}$. The simplified model described in Sect. II predicts a large frequency spacing for the radial mode number and smaller, but comparable, spacing for the poloidal and toroidal mode number increments of $\approx 180 \mathrm{kHz}$ and $\approx 160 \mathrm{kHz}$, respectively. While these are more than twice the observed peak spacing, an explanation may be that the model used for the "well" was greatly simplified and has a high degree of poloidal symmetry not present in the actual well. The poloidal asymmetries present in the actual 
well will introduce further splitting of the eigenfunctions and eigenfrequencies. At present there is no experimental data on the poloidal structure of the modes to constrain the theoretical simulations.

A toroidal array of three high bandwidth Mirnov coils, oriented to measure the vertical component of the magnetic field on the outboard midplane, with relative locations of $0^{\circ}, 20^{\circ}$ and $30^{\circ}$ is used to measure the toroidal wavelength or mode number. A fourth coil, oriented to measure the toroidal component of the magnetic field, is located between the $0^{\circ}$ and $20^{\circ}$ coils. Fourier analysis is used to determine the relative phase shifts of the coherent fluctuations between the coils. In Fig. 8a is shown analysis of a typical mode where, over the array extent of $30^{\circ}$, the phase shift is $\approx 270^{\circ}$ corresponding to a toroidal mode number $\mathrm{n} \approx 9$. The mode propagation is anti-parallel to the injected beam ions, in the $\omega_{*_{\mathrm{e}}}$ direction. The error bars represent the statistical uncertainty in the phase measurement, but do not include possible intrinsic phase errors resulting from small differences in the sensor circuits. In situ calibrations suggest that these relative errors should be less than $10^{\circ}$ at these frequencies.

The amplitude data from all four coils for the same mode is shown in Fig. 8b. The three vertically oriented coils, represented by black circles, show an average magnetic fluctuation amplitude of $8.4 \mu \mathrm{G}$ and the single toroidally oriented coil measures an amplitude of $\approx 10.2 \mu \mathrm{G}$. The toroidal magnetic fluctuations are in phase with the poloidal field fluctuations, which in this coordinate system, indicates that the magnetic fluctuation vector is nearly parallel to the equilibrium field line, i.e., the wave is compressional. A similar analysis of low frequency MHD finds predominantly shearpolarization, as expected. 
In Fig. 9 is shown a comparison of the measured to the predicted CAE frequencies for this experiment. The poloidal mode number is not presently measured and the toroidal mode number is not well defined in many instances, so for simplicity, the predicted frequencies are for the $" \mathrm{~m}=2, \mathrm{n}=6 "$ poloidal eigenmodes, i.e. two wavelengths in the poloidal direction and the measured frequencies are the lowest detected CAE frequency.

The mode drive is predicted to be through a Doppler shifted ion-cyclotron resonance,

$$
\omega=l \omega_{c i}-k_{\theta} V_{d r i f t}-k_{\mathbf{1}} V_{b \|}
$$

where $V_{\text {drift }}$ is the curvature drift velocity of the fast ions and $l$ in this case is unity [13]. The fast ions driving the mode must satisfy this resonance condition and they must also reside in a "bump-on-tail" in the perpendicular energy distribution. There is a further spatial resonance condition such that the optimum mode interaction occurs when the fast ion orbit is comparable to the gradient of the mode structure, $k_{\perp} \rho_{b}>1$. These conditions are investigated with the help of the TRANSP [29] fast ion deposition code.

The TRANSP analysis code calculation of the fast ion distribution function is shown in Fig. 10 for the same shot as in Fig. 4. It should be noted that these computed fast ion distributions do not include effects of the CAE, i.e., the relaxation of the "bump-on-tail" through resonant interaction with the CAE. The TRANSP calculations show that a bump in the perpendicular energy does appear off-axis in NSTX, typically somewhat beyond the half radius. The "bump" can be seen at the full energy of $\approx 58 \mathrm{kV}$ around $\mathrm{V}_{\|} / \mathrm{V}_{\text {beam }} \approx 0.4$ in Fig. 10. Collisional slowing down moves the fast ions predominantly vertically downward (towards lower energy, same pitch-angle) as shown, whereas the CAE extract predominantly perpendicular energy, and would move the fast ions in the 
direction of the black arrow. The other curves overlaid on this figure are discussed below.

The Doppler shifted ion-cyclotron resonance condition constrains the relation between the fast ion parallel velocity and the mode frequency. For simplicity, the parallel wave vector is approximated as $k_{\|}<n / R$, and from Eq. 6 we can define the constraining relationship between the parallel beam velocity and the mode frequency

$$
\frac{V_{b \|}}{V_{b}}>\frac{\omega_{c i} R}{n V_{b}}\left(1-\frac{\omega}{\omega_{c i}}-\frac{k_{\theta} V_{d r i f t}}{\omega_{c i}}\right)
$$

Thus, the fast ion parallel velocity must exceed some threshold level, indicated by the black curve in Fig. 10, to satisfy the resonant condition. Fast ions above and to the right of the black curve in Fig. 10, e.g., those in the "bump-on-tail", have sufficiently large parallel velocity to be resonant with the CAE, those below and to the left are too slow. The curve was calculated for the lowest observed frequency and a toroidal mode number $n=9$; lower mode numbers would shift the curve up and to the right, eventually excluding the fast ion population in the "bump". Higher frequencies and higher toroidal mode numbers, $n>9$, would shift the curve to the left and down. There is considerable uncertainty for some of the parameters in Eq. 7, so this curve should only be considered to indicate that the calculated fast ion population is roughly consistent with the Dopplershifted resonance constraint.

Equation 7 may also be evaluated to find the minimum frequency which satisfies the resonance condition for a given parallel beam velocity. This is shown in Fig. 11 for the full energy beam ion population in the "bump" at $\mathrm{V}_{\|} / \mathrm{V}_{\text {beam }} \approx 0.4$ for the shots in this experiment with unstable CAE. The figure shows the minimum frequency (under the 
above constraints) at which the CAE can fulfill the Doppler shifted resonance condition (Fig. 11, solid circles). For the most part, the observed data satisfies this criterion, but the figure doesn't explain the observed voltage threshold, as the predicted frequency of CAE for the stable shots should also have satisfied this criterion (Fig. 11, open squares).

A further condition on effective resonant drive results from constraints on the effective spatial interaction of the wave with the fast ions [15]. This condition can be approximately described as $1<k_{\perp} \rho_{b}<2$. For the lower limit this constraint may be expressed as $\left(\omega / \omega_{c i}\right)>\left(\alpha V_{A} / V_{\perp b e a m}\right)$ where $\omega \approx \alpha k_{\perp} V_{A}$ is assumed. A reasonable fit to a broad range of data is found with $\alpha \approx 1.3$. This condition introduces a constraint on the ratio of the beam velocity to the Alfvén velocity, or alternatively, a constraint on the minimum mode frequency. This constraint is illustrated on the graph of pitch angle vs. fast ion energy in Fig. 10. The curves labeled "0.93 MHz" and "1.46 $\mathrm{MHz}$ " show the $k_{\perp} \rho_{b}=1$ condition for the two frequencies spanning the observed frequency range. The two curves cover approximately the energy range where a perpendicular "bump-on-tail" exists in the fast ion population. Fast ions near these curves most effectively couple to the CAE.

The upper curve in Fig. 10 corresponds to the lowest frequency CAE in Fig. 4. Lower frequency CAE modes would not have resonant fast ions available to drive them (as the CAE frequency drops, the energy of resonant ions increases). This limit is thus consistent with the observation that CAE activity often "coalesces" at some low frequency limit. The minimum frequency for which energetic ions are spatially resonant with the CAE is $\omega \approx\left(\alpha \mathrm{V}_{\text {Alfvén }} / \mathrm{V}_{\text {beam }}\right) \omega_{\text {ci. }}$. This boundary is shown as the solid curves in Figs. 4, 7 and in Fig. 12. The jump upwards in frequency between 0.2 and 0.22s in Fig. 4 
reflects the change in beam injection voltage from $80 \mathrm{kV}$ to $58 \mathrm{kV}$. Figure 12 compares the predicted to observed minimum CAE frequencies for the data set of Fig. 5.

The fraction of energy in the fast ion distribution available to drive the CAE is relatively small. The NSTX neutral beams inject only about $60 \%$ of the power in the full energy component, the rest is in the half and third energy components, visible in the fast ion distributions shown in Fig. 10. The Doppler-shifted cyclotron resonance takes only perpendicular energy from the fast ions $\left(84 \%\right.$ of the fast ion energy at $\left.\mathrm{V}_{\|} / \mathrm{V}_{\text {beam }}=0.4\right)$ in the "bump-on-tail", which contains roughly $20 \%$ of the full energy ions. Only $\approx 25 \%$ of that energy can be transferred to the mode before the distribution is flattened. Altogether, only a few percent of the beam power deposited at this radius is available to drive the CAE. When the vertical trough where $0.5<\mathrm{V}_{\mathrm{bl}} / \mathrm{V}_{\mathrm{b}}<0.8$ is filled in, there will be no more drive for the modes. The fusion- $\alpha$ population in a reactor may more effectively drive the CAE.

\section{SUMMARY}

The National Spherical Torus Experiment has exhibited unique forms of kinetically driven instabilities during Neutral Beam Injection. In addition to variants of the "fishbone" and TAE modes commonly seen in conventional aspect ratio tokamaks, a much higher frequency instability is commonly seen on NSTX, the compressional Alfvén eigenmode. In this paper it was shown that some of the predictions of a simple theoretical model for the mode dispersion and stability are in good agreement with experimental data. 
The CAE are predicted to exist in a two dimensional potential well localized on the outboard midplane. A numerical code is used to find the eigenvalues of frequency and poloidal wavelength for this well. The code predicts reasonably well the absolute CAE frequency and its' scaling. While the code uses a simplified model for the well, it also uses as input the measured density profile and calculated magnetic field structure of the plasma. It is predicted that the CAE are only weakly localized to the well in the radial direction and show relatively weaker poloidal localization. The CAE generally satisfy the spatial resonance condition, $k_{\perp} \rho>1$.

Both the compressional Alfvén waves and the global Alfvén waves (mixed shear and compressional character) are predicted to be unstable in NSTX [15]. Using an array of four Mirnov coils, with three oriented to measure the poloidal component and one to measure the toroidal component of magnetic field fluctuations, it was shown that these modes indeed have compressional polarization.

The TRANSP code was used to calculate the fast ion distribution and a "bump-ontail" in the perpendicular velocity was found just outside the half-radius. The fast ions in this bump-on-tail were found to satisfy the constraints of the Doppler-shifted ion cyclotron resonance drive condition for the CAE. The spatial resonance condition, $1<k_{\perp} \rho_{b}$, is found to reasonably predict the scaling of the observed minimum CAE frequency.

\section{ACKNOWLEDGEMENTS}

We are grateful to the NSTX team for supporting these experiments. This work supported by U.S. DOE Contract DE-AC02-76CH03073. 


\section{BIBLIOGRAPHY}

[1] G Y Fu and C Z Cheng, Phys. Fluids B 4, 3722 (1992).

[2] M. Ono, S M Kaye, Y-K M Peng, et al., Nucl. Fusion 40, 557 (2000).

[3] L Chen, R B White, M N Rosenbluth, Phys. Rev. Lett. 521122 (1984).

[4] M. P. Gryaznevich, S.E. Sharapov, Nucl. Fusion 40, 907 (2000).

[5] E D Fredrickson, L Chen, R B White, Nucl. Fusion 43, 1258 (2003).

[6] K.L. Wong, R.J. Fonk, S.F. Paul, et al., Phys. Rev. Lett. 66, 1874 (1991).

[7] N N Gorelenkov, C Z Cheng, G Y Fu, S Kaye, and R White, Phys. of Plasmas 7, 1433 (2000).

[8] E D Fredrickson, C Z Cheng, L Chen, D Darrow, N N Gorelenkov, D Johnson, S Kaye, S Kubota, J Menard, R B White, Proc. 29th Eur. Conf. on Plasma Physics and Controlled Fusion, Montreaux, 2002 (European Physical Society, Centre de Recherches en Pysique des Plasmas, CRPP-EPFL, CH1015 Lausanne, Switzerland), Europhysics Conference Abstracts Vol. 26B, P-1.104 (2002).

[9] W W Heidbrink, E Fredrickson, N N Gorelenkov, A W Hyatt, G Kramer and Y Luo, Plasma Physics and Controlled Fusion 45, 983 (2003).

[10] S.M. Mahajan, D.W. Ross, Phys. Fluids 26, 2561 (1983).

[11] B. Coppi, S. Cowley, R. Kulsrud, P. Detragiache, and F. Pegoraro, Phys. Fluids 29, 4060 (1986).

[12] Y.M. Li, S.M. Mahajan, D.W. Ross, Phys. Fluids 30, 2101 (1987).

[13] N.N. Gorelenkov, C.Z. Cheng, Nucl. Fusion 35, 1743 (1995).

[14] E.D. Fredrickson, N. Gorelenkov, C.Z. Cheng, R. Bell, D. Darrow, D. Johnson, S. Kaye, B. LeBlanc, J. Menard, S. Kubota, W. Peebles, Phys. Rev. Lett. 87, 145001 (2001).

[15] N.N. Gorelenkov, E. Fredrickson, E. Belova, C.Z. Cheng, D. Gates, S. Kaye, and R. White, Nucl. Fusion, 43, 228 (2003).

[16] H. Smith, T Fülop, M. Lisak, and D. Anderson, Phys. Plasmas 10, 1437 (2003).

[17] G J Greene, P L Colestock, E D Fredrickson, J C Hosea, K M McGuire, J R Wilson, and K M Young, Proc. $17^{\text {th }}$ Eur. Conf. on Controlled Fusion and Plasma Heating, Amsterdam, 1990 (European Physical Society, Geneva, 1990), Vol. 14B, Part IV, p1540.

[18] S Cauffman, R Majeski, K G McClements, R O Dendy, Nucl. Fusion 35, 1597 (1995).

[19] N N Gorelenkov, C Z Cheng, Phys. Plasmas 2, 1961 (1995). 
[20] T Fülop, Ya. Kolesnichenko, M Lisak, et al., Nucl. Fusion 37, 1281 (1997).

[21] T Fülop, M Lisak, Nucl. Fusion 38, 761 (1998).

[22] E.D. Fredrickson, C.Z. Cheng, D. Darrow, G. Fu, N.N. Gorelenkov, G Kramer, S S Medley, J.

Menard, L. Roquemore, D. Stutman ${ }^{1}$, R. B. White, Phys. of Plasmas 10, 2852 (2003).

[23] N N Gorelenkov, C Z Cheng, E Fredrickson, E Belova, D Gates, S Kaye, G J Kramer, R Nazikian, R B White, Nucl. Fusion 42, 977 (2002).

[24] E D Fredrickson, N N Gorelenkov, C Z Cheng, R. Bell, D. Darrow, D. Gates, D. Johnson, S. Kaye, B. LeBlanc, D. McCune, J. Menard, S. Kubota, W. Peebles, Phys. of Plasmas 9, 2069 (2002).

[25] R. E. Bell, B. P. LeBlanc, C. Bourdelle, et al., , Proc.of 28th Conf. on Controlled Fusion and Plasma Physics, Funchal, 2001, (the European Physical Society, Geneva, 2001) ECA Vol. 25A, p. 1021.

[26] D.W. Johnson, N. Bretz, B. LeBlanc, R. Palladino, D. Long, R. Parsells, Rev. Sci. Instrum. 70, 776 (1999).

[27] L L Lao, H St John, R D Stambaugh, A G Kellman, W P Pfeiffer, Nucl. Fusion 25, 1611 (1985).

[28] S A Sabbagh, S M Kaye, J E Menard, et al., Nucl. Fusion 41, 1601 (2001).

[29] R V Budny, Nucl. Fusion 34, 1247 (1994). 


\section{FIGURE CAPTIONS}

Fig. 1. 2-D shape of CAE well (a) "actual" well shape evaluated with EFIT equilibrium data and eigenvalues as calculated from the (b) simplified well shape (only negative contours are shown). For shot 108841 at $0.259 \mathrm{~s}$ and for the mode numbers $\mathrm{m}=1$ and $\mathrm{n}=6$.

Fig. 2. Numerical calculation of the (a) radial Compressional Alfvén eigenfunction for data in Fig. 5 and (b) effective potential well on outboard midplane vs. normalized minor radius. Radial localization of the mode amplitude is weak.

Fig. 3. Shape of a representative poloidal eigenfunction (for Figs. 1 and 2). Poloidal angle of zero corresponds to outboard midplane. b) Shape of poloidal well.

Fig. 4. Spectragram of Mirnov coil signal showing CAE activity during NBI heating. Curve labeled $" k_{\perp} \rho=1 "$ indicates theoretically predicted lowest unstable frequency.

Fig. 5. Equilbrium from EFIT (a) and density and magnetic field profiles (b and c) used in subsequent analysis. (shot 108841).

Fig. 6. Data set of discharges from experiment to find CAE threshold dependence on 
toroidal field and beam energy. Solid circles are from CAE-unstable discharges, open squares for discharges where no CAE activity was detected.

Fig. 7. Spectrogram of a Mirnov coil signal showing CAE activity. Solid line shows semi-empirical minimum frequency.

Fig. 8. Data from toroidal array of Mirnov coils for same shot as Fig. 7. (a) Relative phase of $1.9 \mathrm{MHz}$ mode at $0.235 \mathrm{~s}$; black square is data from coil oriented to measure toroidal magnetic fluctuations. (b) Amplitude of $1.9 \mathrm{MHz}$ mode, poloidal fluctuation level is $\approx 8.4 \mu \mathrm{G}$, toroidal fluctuation amplitude is $\approx 10.2 \mu \mathrm{G}$.

Fig. 9. Predicted CAE frequency $(m=2)$ vs. lowest observed frequency over range of toroidal field and density.

Fig. 10. Contours of the fast ion distribution function as calculated in TRANSP (logarithmic spacing). The curves labeled "0.93 MHz" and "1.24 MHz" correspond to the condition that $\mathrm{k}_{\perp} \rho=1$ for $\mathrm{CAE}$ at those frequencies (spanning the observed frequency range at this time). The curve labeled "Resonance condtion" corresponds to the Doppler shifted cyclotron resonance condition for $1=1$. The black arrow indicates the trajectory of a fast ion as it loses perpendicular energy to the CAE. 
Fig. 11. Observed CAE frequency (black circles) and predicted CAE frequency for stable shots (open black squares) vs. the minimum frequency for which the Doppler shifted resonance condition could be met.

Fig. 12. Mirnov spectrogram showing CAE coalescence at predicted minimum frequency (curve labeled " $k_{\perp} \rho=1 "$ ).

Fig. 13. Scaling of approximate minimum CAE frequency vs. model prediction, $\gamma=1$. 


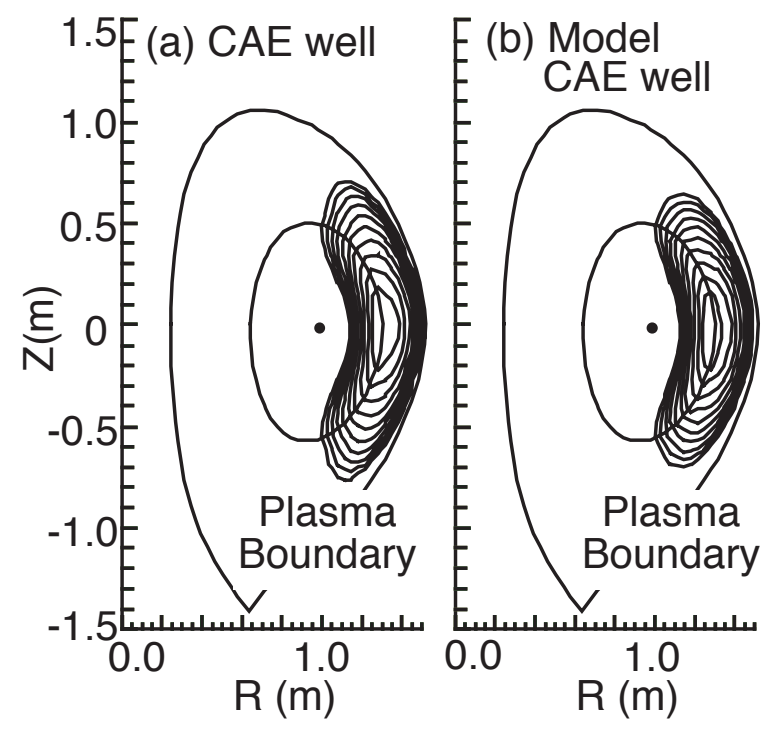




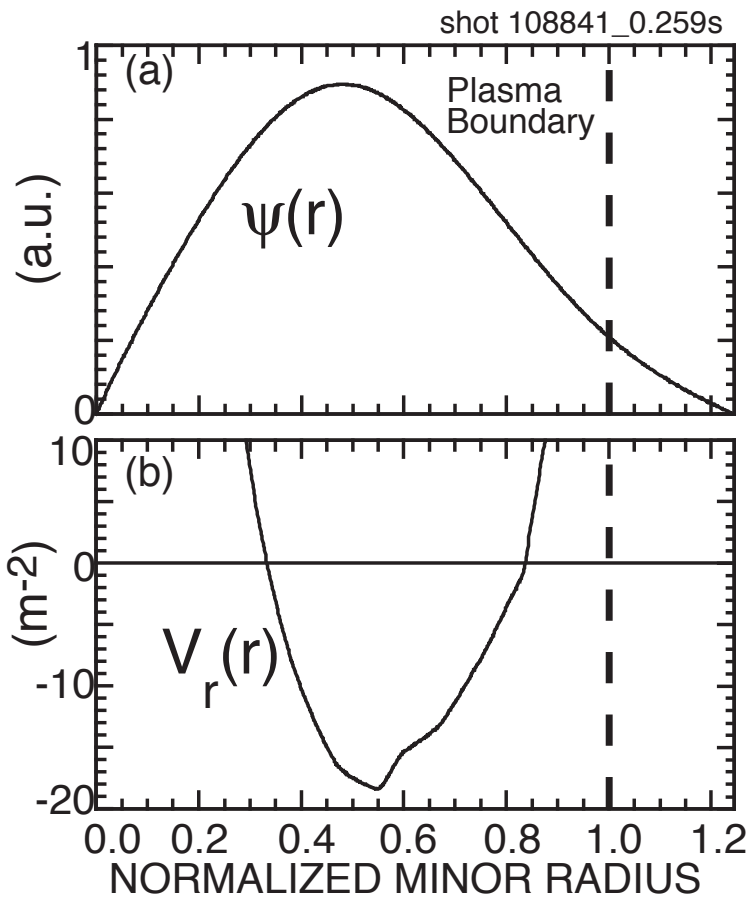




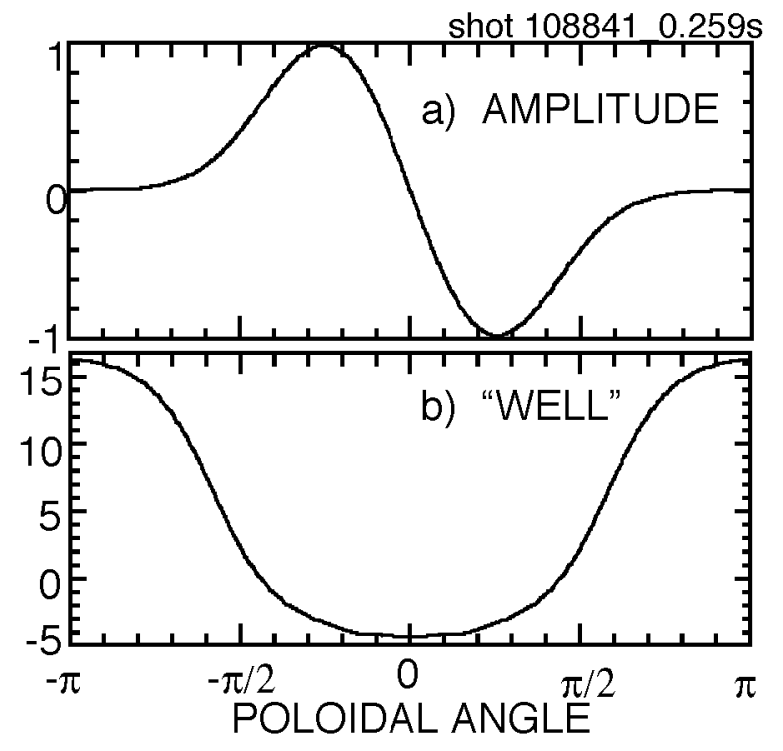


108841

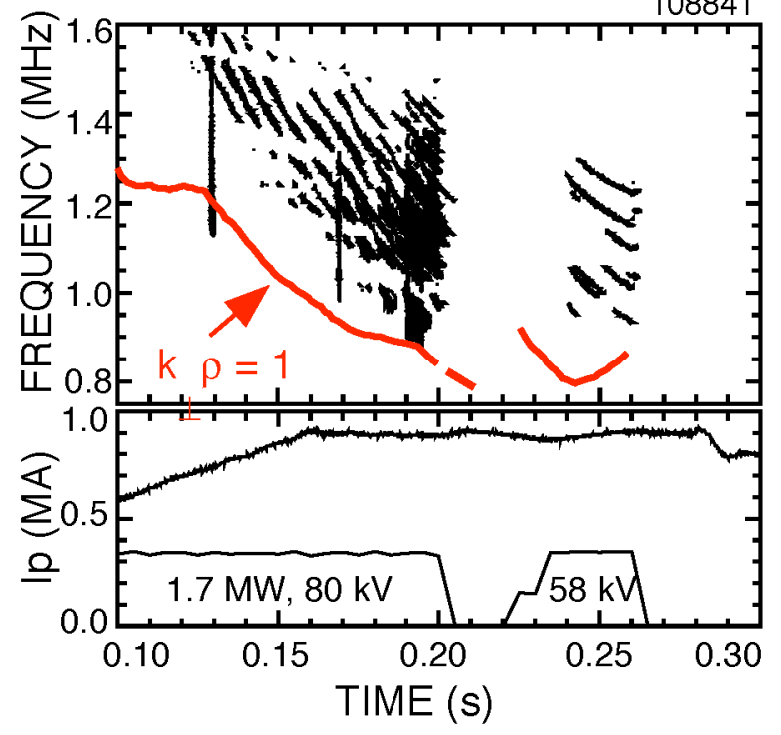




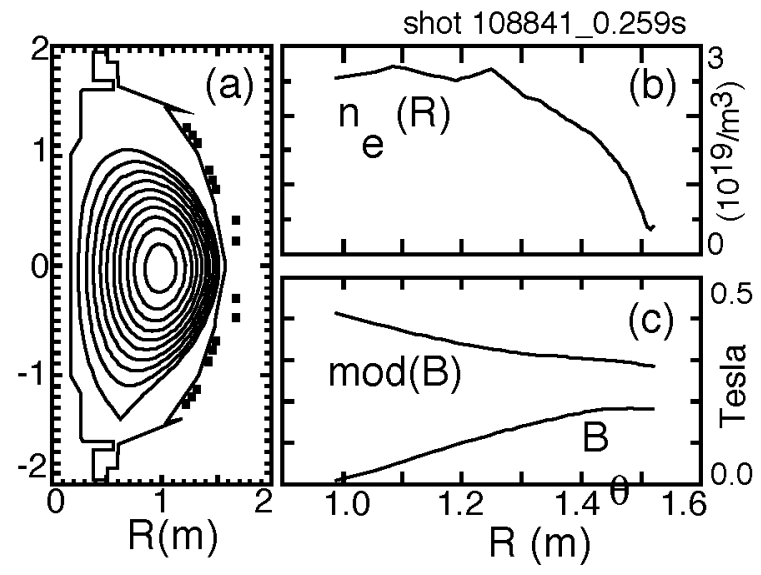




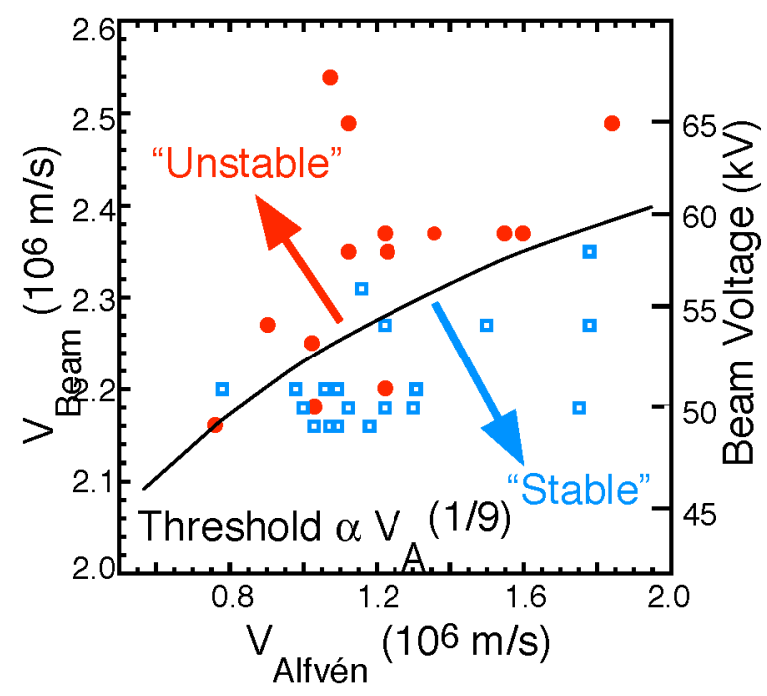




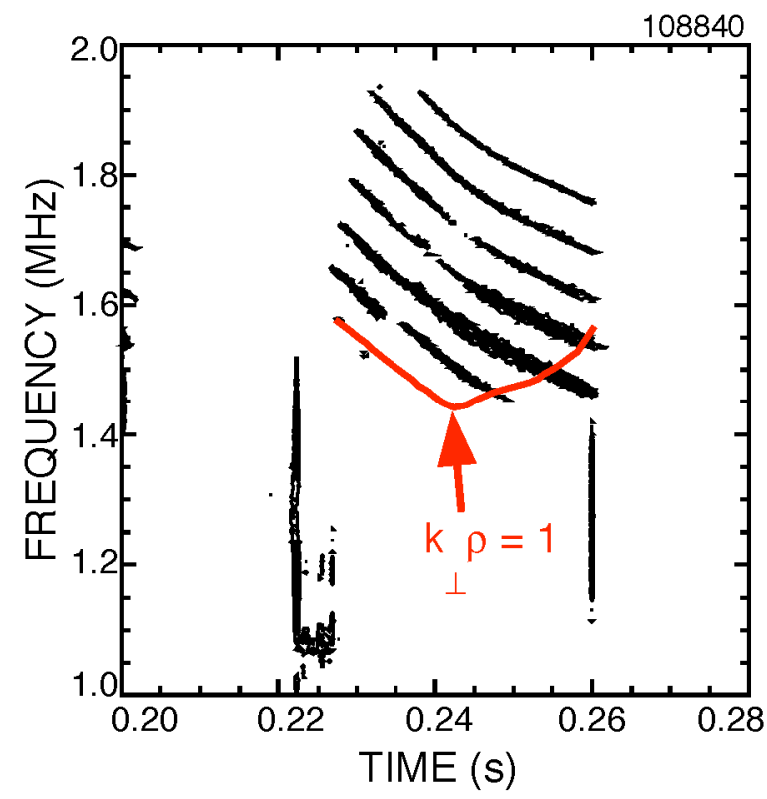


108840_0.235s_1.889MHz

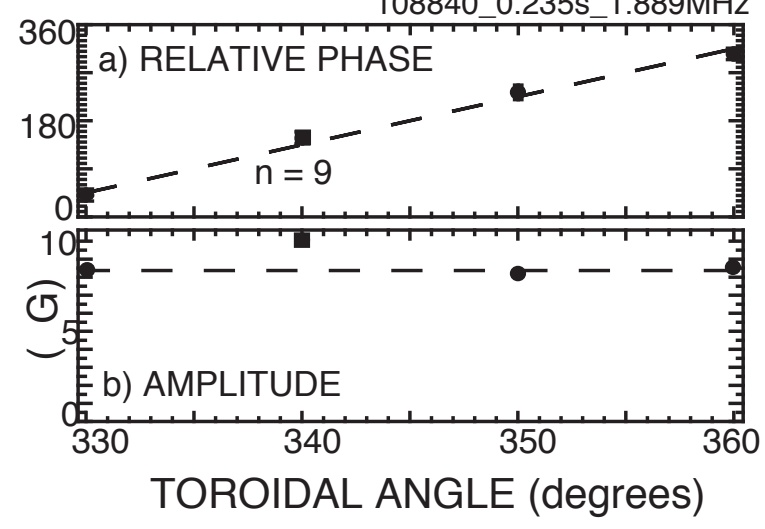




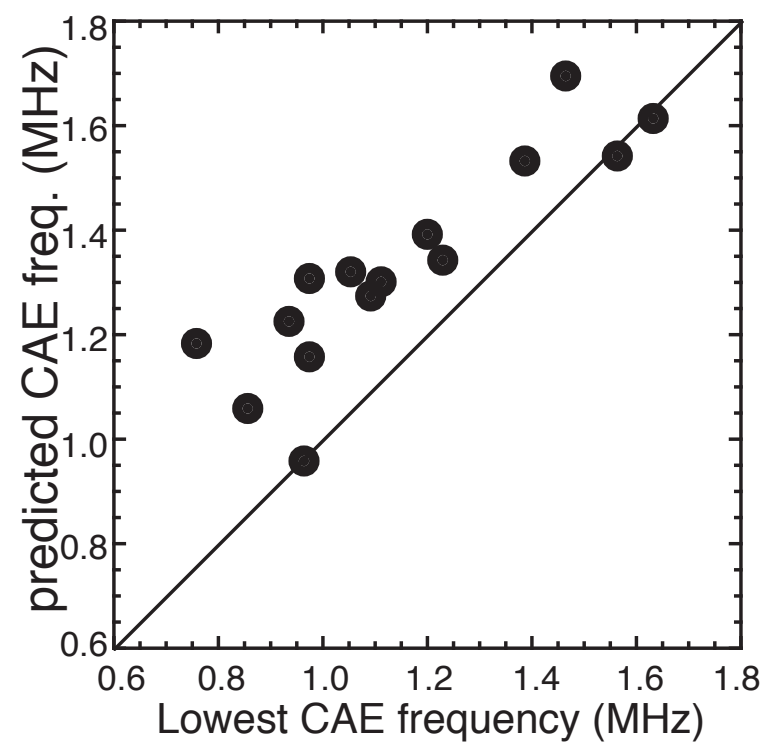




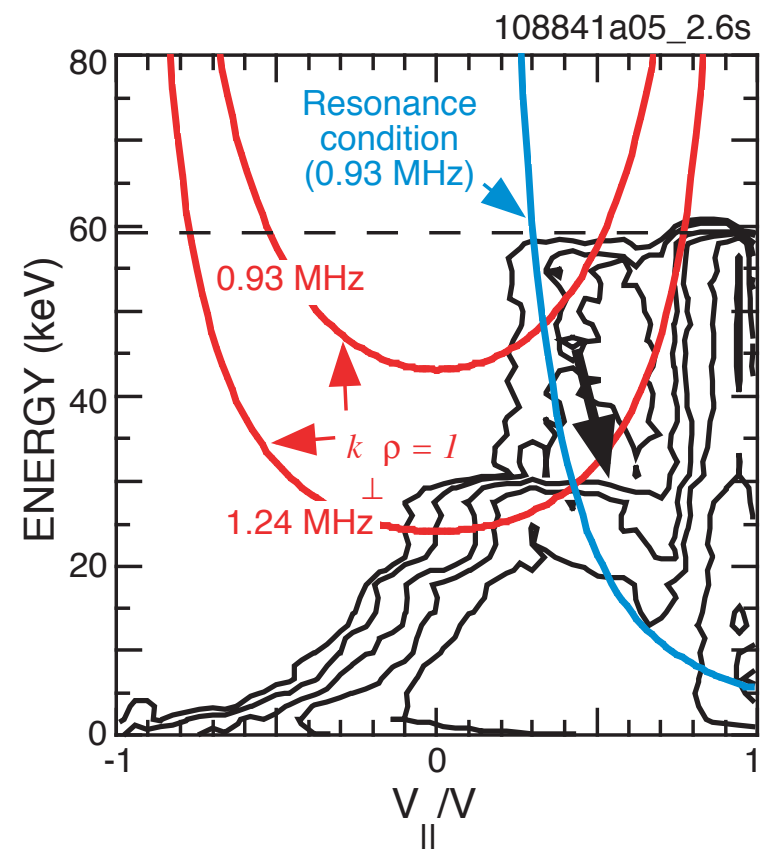




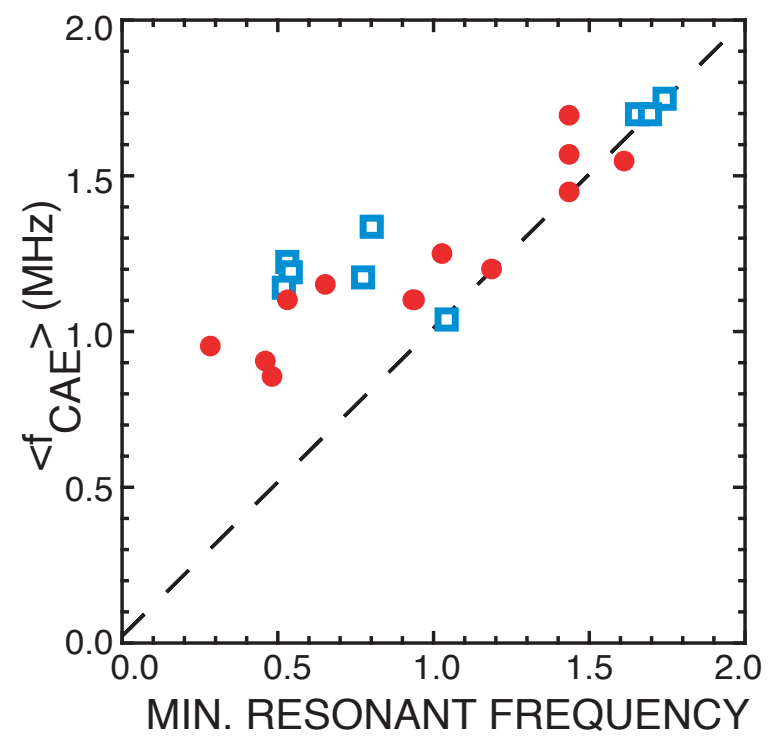




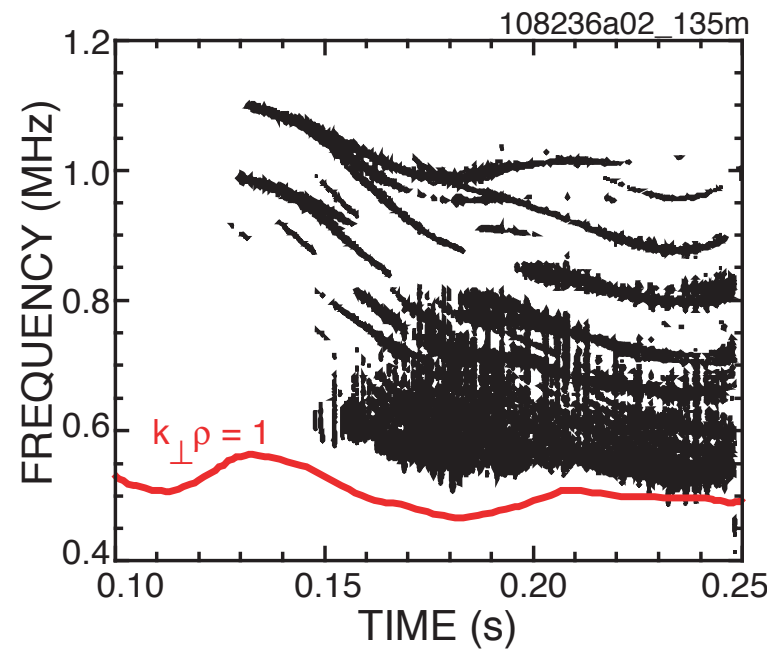




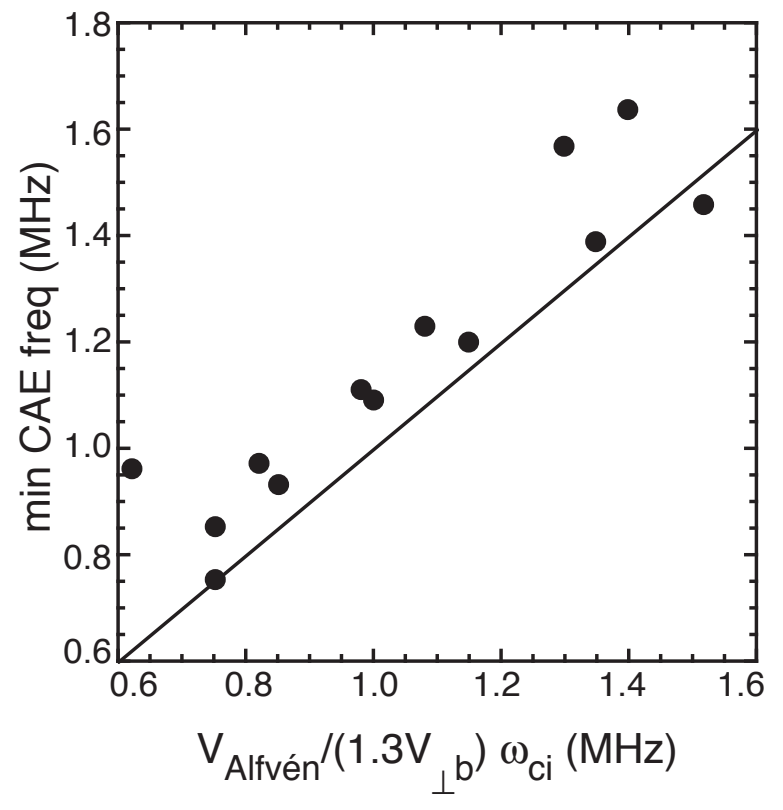




\section{External Distribution}

Plasma Research Laboratory, Australian National University, Australia

Professor I.R. Jones, Flinders University, Australia

Professor João Canalle, Instituto de Fisica DEQ/IF - UERJ, Brazil

Mr. Gerson O. Ludwig, Instituto Nacional de Pesquisas, Brazil

Dr. P.H. Sakanaka, Instituto Fisica, Brazil

The Librarian, Culham Laboratory, England

Mrs. S.A. Hutchinson, JET Library, England

Professor M.N. Bussac, Ecole Polytechnique, France

Librarian, Max-Planck-Institut für Plasmaphysik, Germany

Jolan Moldvai, Reports Library, Hungarian Academy of Sciences, Central Research Institute for Physics, Hungary

Dr. P. Kaw, Institute for Plasma Research, India

Ms. P.J. Pathak, Librarian, Institute for Plasma Research, India

Ms. Clelia De Palo, Associazione EURATOM-ENEA, Italy

Dr. G. Grosso, Instituto di Fisica del Plasma, Italy

Librarian, Naka Fusion Research Establishment, JAERI, Japan

Library, Laboratory for Complex Energy Processes, Institute for Advanced Study, Kyoto University, Japan

Research Information Center, National Institute for Fusion Science, Japan

Dr. O. Mitarai, Kyushu Tokai University, Japan

Dr. Jiangang Li, Institute of Plasma Physics, Chinese Academy of Sciences, People's Republic of China

Professor Yuping Huo, School of Physical Science and Technology, People's Republic of China

Library, Academia Sinica, Institute of Plasma Physics, People's Republic of China

Librarian, Institute of Physics, Chinese Academy of Sciences, People's Republic of China

Dr. S. Mirnov, TRINITI, Troitsk, Russian Federation, Russia

Dr. V.S. Strelkov, Kurchatov Institute, Russian Federation, Russia

Professor Peter Lukac, Katedra Fyziky Plazmy MFF UK, Mlynska dolina F-2, Komenskeho Univerzita, SK-842 15 Bratislava, Slovakia

Dr. G.S. Lee, Korea Basic Science Institute, South Korea

Institute for Plasma Research, University of Maryland, USA

Librarian, Fusion Energy Division, Oak Ridge National Laboratory, USA

Librarian, Institute of Fusion Studies, University of Texas, USA

Librarian, Magnetic Fusion Program, Lawrence Livermore National Laboratory, USA

Library, General Atomics, USA

Plasma Physics Group, Fusion Energy Research Program, University of California at San Diego, USA

Plasma Physics Library, Columbia University, USA

Alkesh Punjabi, Center for Fusion Research and Training, Hampton University, USA

Dr. W.M. Stacey, Fusion Research Center, Georgia Institute of Technology, USA

Dr. John Willis, U.S. Department of Energy, Office of Fusion Energy Sciences, USA

Mr. Paul H. Wright, Indianapolis, Indiana, USA 
The Princeton Plasma Physics Laboratory is operated by Princeton University under contract with the U.S. Department of Energy.

\author{
Information Services \\ Princeton Plasma Physics Laboratory \\ P.O. Box 451 \\ Princeton, NJ 08543
}

Phone: 609-243-2750

Fax: 609-243-2751

e-mail: pppl_info@pppl.gov

Internet Address: http://www.pppl.gov 